\title{
Tensions in neutrino-nucleus modeling
}

\section{Joanna E. Sobczyk*}

Instituto de Física Corpuscular (IFIC), Centro Mixto CSIC-Universidad de Valencia, E-46071

Valencia (Spain)

E-mail: jsobczykeific.uv.es

\section{Juan Nieves}

Instituto de Física Corpuscular (IFIC), Centro Mixto CSIC-Universidad de Valencia, E-46071

Valencia (Spain)

E-mail: jmnieves@ific.uv.es

\begin{abstract}
Neutrino-nucleus modeling occupies an important place in the neutrino oscillation programme, since it is essential for the precise reconstruction of neutrino energy. Due to nuclear effects, theoretical uncertainties stemming from neutrino-nucleus cross sections largely contribute to the overall systematic errors of oscillation experiments.

We present an overview of challenges which we encounter in this field, paying attention to the ones which are motivated by the Monte Carlo (MC) generator studies. Next, we make a survey of various theoretical models, sketching the main differences between them. We organize the discussion according to the physical mechanisms which give contributions to the total cross section of the neutrino-nucleus scattering, starting from the giant resonances, through the QE excitation, multinucleon knockout, pion production, up to the DIS region.
\end{abstract}

The 21st international workshop on neutrinos from accelerators (NuFact2019)

August 26 - August 31, 2019

Daegu, Korea

${ }^{*}$ Speaker. 


\section{Introduction}

We are entering the era of high-precision neutrino oscillation experiments, which - in the case of DUNE [1] and Tokai to Hyper-Kamiokande (T2HK) [2] - for the first time in history will reach such a high rate of observed events that the statistical errors will diminish considerably and they will no longer dominate in the overall experimental uncertainties. As a result, the control over systematic errors will play a crucial role in the success of experimental programs. The two experiment mentioned above will primarily aim at measuring the $\mathrm{CP}$ violating phase in the weak sector. Moreover, they will allow for a much wider research program able to detect the signs of New Physics.

One of the main sources of systematic uncertainties are neutrino-nucleus cross sections. Since neutrinos are not detected directly, we are forced to reconstruct their energy (crucial to obtain the oscillation probability pattern) by observing the outgoing particles in each event. Since the processes take place on nuclei (not on free nucleons), the nuclear effects have to be taken into account. In fact, they are mostly responsible for high theoretical uncertainties.
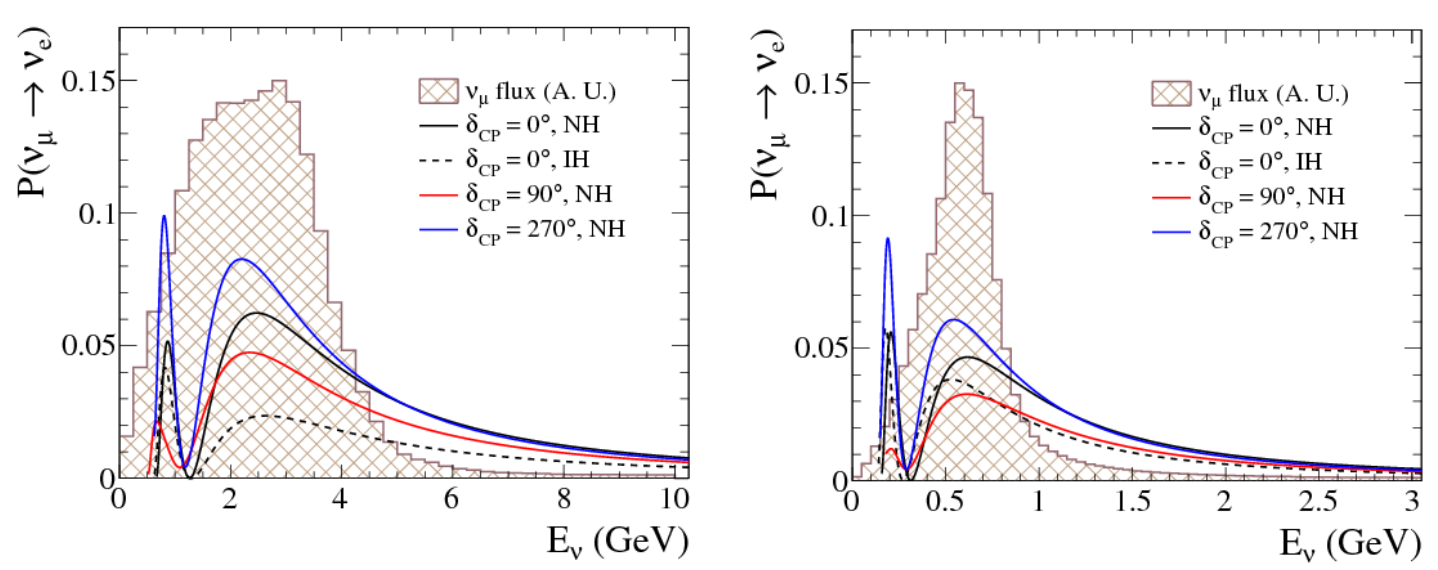

Figure 1: Fluxes and oscillation probability patterns $P\left(v_{\mu} \rightarrow v_{e}\right)$ in the far detector for various values of the $\mathrm{CP}$ violating phase and mass hierarchy (either normal, $\mathrm{NH}$, or inverted, $\mathrm{IH}$ ). In the right panel we show predictions for DUNE (distance to the detector $1300 \mathrm{~km})$, on the left panel for T2HK $(295 \mathrm{~km})$ experiments. Figure taken from Ref. [3].

The dependence of the neutrino oscillation pattern on the CP violating phase is shown in Fig. 1 for DUNE and T2HK experiments. Measuring with high resolution both the neutrino energy and the total cross section is essential to precisely predict the shape of oscillation pattern curve and subsequently the value of the $\mathrm{CP}$ phase. Depending on the technology and particular experimental analysis, various strategies can be used to achieve this goal. In fact, DUNE and T2HK will use complimentary methods (calorymetric and kinematical) [4]. In both cases, the analyses strongly rely on the predictions given by the Monte Carlo (MC) event generators, which implement theoretical nuclear models.

It is also worth mentioning that the exposure time needed to detect the $\mathrm{CP}$ violating phase diminishes considerably when the systematic uncertainties are reduced. In Fig. 2 we show the 
predicted exposure time as a function of three different values of systematic errors (apart from the flux uncertainty set here to $5 \%$ ). Going from $3 \%$ to $1 \%$ systematic errors would lead to around $30 \%$ shorter time needed to detect the $\mathrm{CP}$ violation.

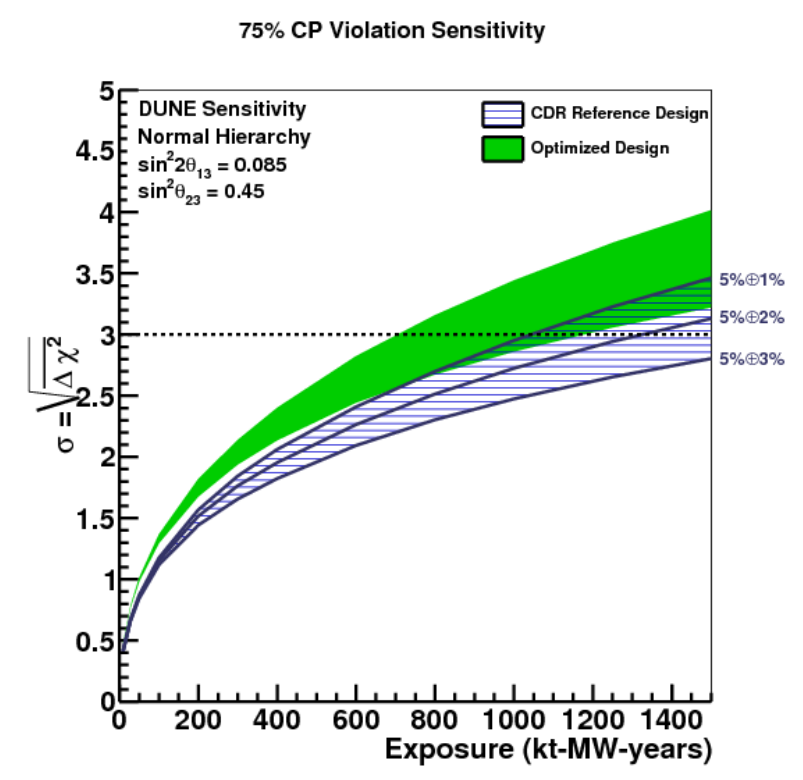

Figure 2: DUNE sensitivity for the measurement of the CP violating phase as a function of exposure (see the text for a more detailed description). Figure taken from Ref. [5].

One can hope to partially get rid of uncertainties - coming from the nuclear effects and the neutrino flux - using the measurements obtained in the near detectors. The analysis is non trivial, since it involves various sources of errors. Nevertheless, the information from the near detector can constrain systematics in the far detector. Still, the information about neutrino-nucleus interactions is needed for the analyses in both of them, since the flux of neutrinos is different due to oscillations, and the detectors are usually built of different materials, so nuclear effects are not necessarily the same.

In the view of these various motivations, we present a status of the models for neutrino-nucleus interactions. In Sec. 2 we list the main practical challenges which emerge from the experimental and the MC generators studies. The theoretical perspective on this problem is briefly sketched in Sec. 3. Next, in Sec. 4, we present a survey comprised of the most important physical mechanisms which contribute to the total cross section.

\section{Challenges}

Taking into account the challenges coming from the experimental studies and from Monte Carlo event generators, one can propose a list of desired features required from the theoretical models for neutrino-nucleus interactions implemented in the MCs.

- Since neutrino fluxes are not monochromatic, we need models which would give predictions for neutrino-nucleus interactions in the whole range of energies comprised between tens of $\mathrm{MeV}$ up to few $\mathrm{GeV}$. 
- The models should work for a variety of nuclear species, since the detectors are made of different nuclei (carbon, oxygen, argon, etc.).

- We are not only interested in the inclusive cross sections, but it is also important to know the predictions for the distributions of outgoing hadrons (as an input to the MC cascade).

- We would like to control the theoretical uncertainties and - in the case of MC generators have some free parameters of the models to tune the predictions.

- For the practical purposes, the implemented models in the MC generators should allow for a fast numerical computation.

- The validation of theoretical models is of primary importance. They should be compared with available data (e.g. for electron scattering processes whose measurements are much more exact than for the processes triggered by neutrinos).

\section{Theoretical perspective}

The modeling of neutrino-nucleus interactions is a challenging problem. Various reaction mechanisms (see Sec. 4) give contribution to the response of the nuclear system, depending on the energy-momentum transferred to the nucleus. In the most general terms, this many-body problem can be defined in terms of $|i\rangle,|f\rangle$ - initial and final nuclear states, and the transition operator (here we will consider the charge-current transition):

$$
j_{c c}^{\mu}=\bar{u} \gamma^{\mu}\left(1-\gamma^{5}\right)\left(d \cos \theta_{C}+s \sin \theta_{C}\right)
$$

with quark fields $u, d, s$ and the Cabbibo angle $\theta_{C}$. The inclusive cross section is proportional to the contraction of the leptonic and hadronic tensors, $d \sigma /\left(d E_{k^{\prime}} d \Omega_{k^{\prime}}\right) \propto L_{\mu \nu} W^{\mu v}$,

$$
W^{\mu v} \propto\left(J^{\mu}\right)^{\dagger} J^{v}, \quad J^{\mu}=\left\langle f\left|j_{c c}^{\mu}\right| i\right\rangle, \quad L^{\mu v} \propto \operatorname{Tr}\left[k \gamma^{\mu}\left(1-\gamma^{5}\right)\left(k^{\prime}+m_{l}\right) \gamma^{v}\left(1-\gamma^{5}\right)\right],
$$

where $k, k^{\prime}$ are incoming and outgoing lepton momenta and $m_{l}$ is the outgoing lepton mass. The most accurate description of the nuclear system (and also of its responses) available nowadays, is given by ab-initio calculations, which use the state-of-the-art nucleon-nucleon potentials to solve numerically the many-body problem [6]. Still, these approaches are limited due to the huge computational cost. Furthermore, they are not able to address the relativistic effects, since the dynamics of the system is described in terms of a nonrelativistic Hamiltonian. Moreover, only the inclusive cross sections can be obtained within these formalisms. With all these restrictions, certainly they can serve as a benchmark for more approximated (but used in practical situations) methods.

In the neutrino-nucleus studies, various approximations were proposed by different theory groups. The initial nuclear state can be reduced to a system of independent particles using a meanfield picture in which each of them feels the same averaged field produced by the remaining particles of the system. This approach is followed by the Ghent group [7] which uses an effective Skyrme potential with parameters fitted to reproduce low-energy excitations of spherical nuclei.

Another approach consists in calculating the hole spectral function, which gives the probability distribution of finding an initial nucleon depending on its momentum and energy. In the formalism 
of Ref. [8], the hole spectral function is obtained as a sum of two contributions. The dominating one comes from the shell-model (mean-field) calculations, while the other one, which corresponds to the region of high energy-momentum, is obtained using the local density approximation (LDA) and ab-initio Correlation Basis Function results for nuclear matter. The Valencia group also uses the spectral function formalism [9]. However, in this case, the model is semi-phenomenological and it starts from the elastic nucleon-nucleon scattering data as an input, with some in-medium modifications. The calculations for a given nucleus are done assuming the LDA. The virtue of this model is the fact that both the initial and final nucleons can be described by means of the same formalism (this is true also in the case of the Ghent formalism). Another scheme, SuSAv2, was derived from a phenomenological approach SuSA, which uses as an input the experimental scaling function obtained from the analysis of electromagnetic responses and uses them to predict the results for the electroweak processes. SuSAv2 combines these advocated scaling properties with results computed for isovector and isoscalar response functions within the relativistic meanfield approach in the region of medium and high energy transfers [10]. GiBUU [11] describes the nuclear system using the LDA. The nucleons are submerged into a momentum and position (density) dependent potential (obtained from the heavy-ion collision studies).

Even though various theoretical studies are available, the most widely used approach for the nuclear effects of the initial state in the MC generators accounts only for Pauli-blocking and an average binding energy (Fermi gas model). This oversimplified picture of the nuclear dynamics is well-known for its poor performance [12].

The nuclear effects of the initial state are only the first step of the problem. Next, we have to properly describe the dynamics of a particular process, and the nuclear effects affecting the final particles. In the following section we will make a survey of various reaction mechanisms, leaving apart, however, the details of how the particles produced in the weak vertex afterwards are propagated through the nucleus (this is simulated by the Monte Carlo cascade implemented in the event generators).

\section{Physical mechanisms}

In Fig. 3 we present a schematic picture of the neutrino-nucleus differential cross section as a function of the energy transfer. Various physical mechanisms are shown to dominate at different energies. The figure clearly shows how challenging it is to develop a consistent framework which would account for such a variety of interaction channels. The resolution of the electroweak probe ranges from very low energies, for which the structure of the nucleus cannot be described in terms of independent nucleons, up to high values in which the scattering takes place on quarks. In the following subsections we will describe the main challenges appearing in each region, and the approaches developed by various theoretical groups.

\subsection{Giant resonances}

In this region of low energy-momentum transfers, the long-range correlations (RPA) play an important role and collective modes of the nucleus are excited. The resolution of the gauge boson is so low that it does not "see" separate nucleons. Therefore, this mechanism goes beyond the 


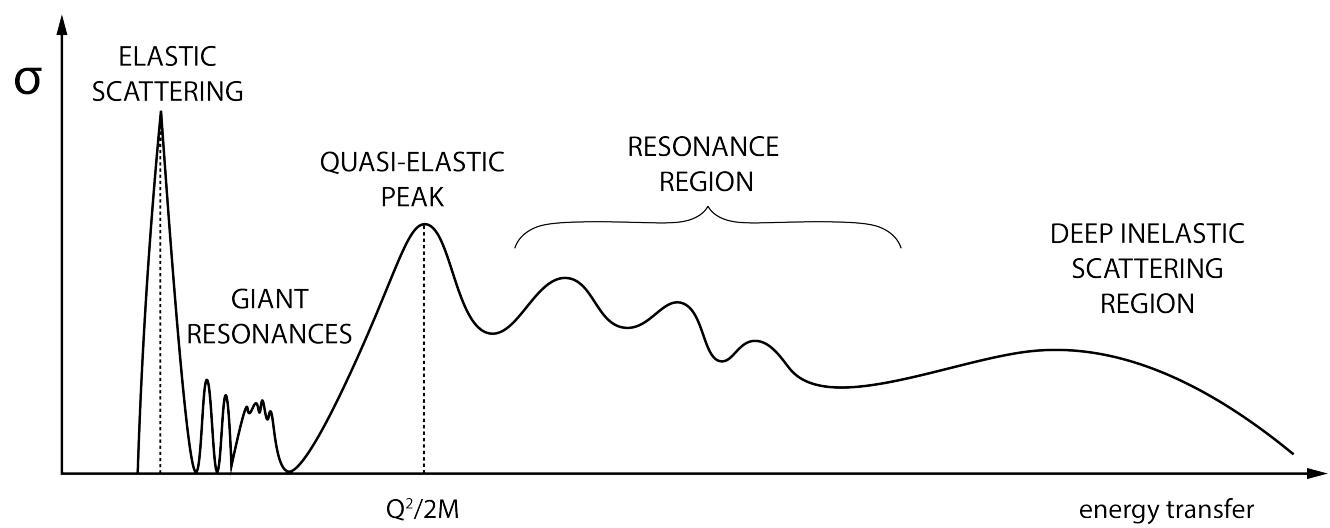

Figure 3: The spectrum of neutrino-nucleus scattering as a function of energy transfer. The most important physical mechanisms contributing to the cross section are indicated.

capabilities of the mean-field description (and it cannot be addressed by e.g. spectral function formalisms).

The Ghent group has developed a continuum RPA approach to describe this region [7]. They use the same residual interaction as the one which is employed in their mean-field calculation. The agreement with the available electron scattering inclusive data is quite good, as can be observed in Fig. 5 of Ref. [7]. The Valencia group also accounts for the RPA effects. In their approach, however, which is based on the LDA, only the inclusive cross section can be predicted, without details of the spectrum of the resonances. The results have been compared against the data for muon and pion radiative captures $[9,13]$. This approach (based on an effective Landau-Migdal potential) is very similar to the one which was proposed by the Lyon group [14].

\subsection{Quasielastic mechanism}

For momentum transfers of the order of $\gtrsim 200-300 \mathrm{MeV}$, which correspond to the internucleon distances in the nucleus, the electroweak probe in a good approximation scatters off a single nucleon. The quasielastic (QE) mechanism corresponds to a situation in which a single nucleon is produced in the primary weak vertex. In the case of neutrino-nucleon scattering, this would lead to a condition $q^{0}=-q^{2} / 2 M$ (with the energy-momentum transfer $q=\left(q^{0}, \vec{q}\right)$ ). In the nucleus, this relation is modified through nuclear effects affecting both the initial and final states, and so the QE peak is shifted. It is also spread due to the nucleons motion.

Various models for the initial nuclear state introduced in the previous section (i.e. approximations based on the mean-field, spectral functions or in-medium potential) should also provide a proper description of the outgoing nucleon which "feels" the nuclear medium. Within the Ghent model, both (initial and final) nucleons are described in the same mean-field picture. This consistent framework is, nevertheless, nonrelativistic. The authors of the spectral function formalism of Ref. [15], propose to account for the final state interactions using a convolution scheme, introducing an optical potential and nuclear transparencies. The Valencia model uses the same spectral functions for the particle state or - in the relativistic regime - treats it as a free particle. GiBUU's potential is relativistic, and as such can be safely used for the outgoing nucleons in the relativistic regime. 
All the above mentioned models were compared with a broad selection of inclusive electron scattering data [12] and have proved to work fairly well for a variety of kinematic setups. This conclusion cannot be drawn for the Fermi gas models, which are unable to properly predict the shape and position of the QE peak.

\subsection{Pion production}

Pion production is an important channel from the point of view of neutrino oscillation studies since a great part of the total cross section in many experiments is produced through this process. In a good approximation, the interaction takes place on a single bound nucleon, and a pion-nucleon pair is produced in the final state. The dynamics of this reaction is much more complicated than in the case of the QE mechanism. Depending on the invariant mass of the hadron system of outgoing particles and the isospin channel, the interaction is dominated by various resonances. Moreover, the background terms are also present and might play an important role. At intermediate energies, the bulk of the strength is driven by the $\Delta(1230)$ excitation. Still, a tower of heavier resonances should be included to describe the higher energy transfer region. This, however, introduces further complications. First of all, the axial form factors of the resonances are not known. Second of all, the estimates of the non-resonant background at low energies derived from chiral perturbation theory should be expanded to include further terms, and other degrees of freedom than pions also need to be incorporated. Importantly, the theory should be unitarized.

At the single nucleon level, one of the most accurate descriptions of electroweak pion production was obtained within the DCC (dynamical coupled channel) model [16]. It incorporates twoand three-body unitarity and describes hadron states up to invariant mass $W<2 \mathrm{GeV}$. Another widely used model of the pion production was proposed by the Valencia group, described in a series of publications $[17,18,19]$. It was designed to describe the vicinity of the $\Delta$ peak (invariant mass $W<1.4 \mathrm{GeV}$ ) and incorporates a partial unitarization procedure through the inclusion of Watson phases. Recently a detailed comparison between this model and the DCC approach has been performed [20]. It shows quite a good agreement in the $\Delta$ region for various observables. The model of Ref. [17] has also become a starting point for an approach presented in Ref. [21], in which it was extended to high-energies using a Regge approach. In the case of the GiBUU approach, the pion production model for low invariant masses is based on the Valencia model, while for high energies, a phenomenological background is included. Still, MC generators widely use the Rein-Sehgal model that was proposed in Ref. [22], and that does not account for any non-resonant background contributions.

\subsection{Multinucleon knock-out}

This is an additional dynamical mechanism that gives contribution to the nuclear response in the energy range comprised between the $\mathrm{QE}$ and $\Delta$ peaks. It corresponds to the situation in which the scattering takes place on two correlated nucleons, and as a result a pair of nucleons is created in the final state ( $2 \mathrm{p} 2 \mathrm{~h})$. Also a process with more than two correlated nucleons might occur, however its contribution is much less significant. This $2 \mathrm{p} 2 \mathrm{~h}$ reaction mechanism is a many-body effect and it requires a careful treatment of the nucleon-nucleon interaction in the nuclear medium. 5 Various theoretical models have been proposed [14, 23, 24, 25]. They are predominantly based on the Fermi gas distribution of the initial nucleons (with the exception of Ref. [25] in which the hole spectral 
function is used for their description). In Ref. [24] the correlations between nucleons is based on only one pion exchange, while in Ref. [23] an effective in-medium potential is used which goes beyond the latter mechanism, including both longitudinal and transverse channels of interaction. GiBUU uses a phenomenological fit [26].

In some kinematical setups there might be a tension between various approaches. It is also worth mentioning that it is challenging to draw conclusions about their compatibility with the experimental measurements. One has to include all the dynamical channels: QE, pion production and $2 \mathrm{p} 2 \mathrm{~h}$, to compare with the available high-quality electron scattering data. Therefore, the theoretical uncertainties of all the channels have to be taken into account.

\subsection{SIS and DIS regions}

When the invariant mass grows, the two pion production channel opens and starts dominating over the reaction with just one pion in the final state. Eventually we enter a region in which the gauge boson wavelength is so short that it probes the structure of nucleons. The scattering takes place on the quarks bound in nucleons. This deep inelastic scattering (DIS) region will play an important role in DUNE experiment due to its flux peaking around $E_{v}=3 \mathrm{GeV}$. The transition region between resonance excitations and DIS - also called as Shallow Inelastic Scattering (SIS) is particularly challenging to address since the degrees of freedom change from hadrons to (bound) quarks (in the electron-nucleon scattering a so-called quark-hadron duality was seen [27]). Recently, a dedicated workshop on SIS and DIS in neutrino scattering was organized [28], and we refer the reader there for further developments in this topic.

\section{Conclusions}

Certainly, a lot of work has been done in the last years to address the problem of neutrinonucleus interactions. This interest was triggered by the experimental needs for theoretical models able to describe a large spectrum of physical mechanisms which give raise to the total cross section, for neutrino energies ranging from tens of $\mathrm{MeV}$ to few $\mathrm{GeV}$.

Even though the theoretical studies are quite advanced - mostly drawing on experience from the electron scattering processes - there are still open questions, tensions between some theoretical predictions, and an ongoing discussion about various issues e.g. a role played by the multinucleon knock-out mechanism. The theoretical predictions were confronted with a variety of experimental data, although the emphasis has been put on the comparisons with inclusive cross sections, while in the MC generators we are also interested in the outgoing hadrons distributions. These detailed comparisons serve as a much more demanding test which can give further insights into the limitations of theoretical approaches.

A special care has to be taken to ensure the consistency of the models for various mechanisms used in MC generators. Another challenge would be to fill the gap between theoretical developments and models - quite often phenomenological or oversimplified - which are actually adopted in the experimental studies.

\section{References}

[1] http://www.dunescience.org. 
[2] K. Abe et al. [Hyper-Kamiokande Working Group], arXiv:1412.4673 [physics.ins-det].

[3] M. V. Diwan, V. Galymov, X. Qian and A. Rubbia, Ann. Rev. Nucl. Part. Sci. 66 (2016) 47 doi:10.1146/annurev-nucl-102014-021939 [arXiv:1608.06237 [hep-ex]].

[4] L. Alvarez-Ruso et al., Prog. Part. Nucl. Phys. 100 (2018) 1 doi:10.1016/j.ppnp.2018.01.006 [arXiv:1706.03621 [hep-ph]].

[5] R. Acciarri et al. [DUNE Collaboration], arXiv:1512.06148 [physics.ins-det].

[6] A. Lovato, S. Gandolfi, J. Carlson, S. C. Pieper and R. Schiavilla, Phys. Rev. Lett. 117 (2016) no.8, 082501 doi:10.1103/PhysRevLett.117.082501 [arXiv:1605.00248 [nucl-th]].

[7] V. Pandey, N. Jachowicz, T. Van Cuyck, J. Ryckebusch and M. Martini, Phys. Rev. C 92 (2015) no.2, 024606 doi:10.1103/PhysRevC.92.024606 [arXiv:1412.4624 [nucl-th]].

[8] O. Benhar, A. Fabrocini, S. Fantoni and I. Sick, Nucl. Phys. A 579 (1994) 493. doi:10.1016/0375-9474(94)90920-2

[9] J. Nieves and J. E. Sobczyk, Annals Phys. 383 (2017) 455 doi:10.1016/j.aop.2017.06.002 [arXiv:1701.03628 [nucl-th]].

[10] R. Gonzaléz-Jiménez, G. D. Megias, M. B. Barbaro, J. A. Caballero and T. W. Donnelly, Phys. Rev. C 90 (2014) no.3, 035501 doi:10.1103/PhysRevC.90.035501 [arXiv:1407.8346 [nucl-th]].

[11] O. Buss et al., Phys. Rept. 512 (2012) 1 doi:10.1016/j.physrep.2011.12.001 [arXiv:1106.1344 [hep-ph]].

[12] J. E. Sobczyk, Phys. Rev. C 96 (2017) no.4, 045501 doi:10.1103/PhysRevC.96.045501 [arXiv:1706.06739 [nucl-th]].

[13] J. Nieves, J. E. Amaro and M. Valverde, Phys. Rev. C 70 (2004) 055503 Erratum: [Phys. Rev. C 72 (2005) 019902] doi:10.1103/PhysRevC.70.055503, 10.1103/PhysRevC.72.019902 [nucl-th/0408005].

[14] M. Martini, M. Ericson, G. Chanfray and J. Marteau, Phys. Rev. C 80 (2009) 065501 doi:10.1103/PhysRevC.80.065501 [arXiv:0910.2622 [nucl-th]].

[15] A. M. Ankowski, O. Benhar and M. Sakuda, Phys. Rev. D 91 (2015) no.3, 033005 doi:10.1103/PhysRevD.91.033005 [arXiv:1404.5687 [nucl-th]].

[16] H. Kamano, S. X. Nakamura, T.-S. H. Lee and T. Sato, Phys. Rev. C 88 (2013) no.3, 035209 doi:10.1103/PhysRevC.88.035209 [arXiv:1305.4351 [nucl-th]].

[17] E. Hernandez, J. Nieves and M. Valverde, Phys. Rev. D 76 (2007) 033005 doi:10.1103/PhysRevD.76.033005 [hep-ph/0701149].

[18] L. Alvarez-Ruso, E. Hernández, J. Nieves and M. J. Vicente Vacas, Phys. Rev. D 93 (2016) no.1, 014016 doi:10.1103/PhysRevD.93.014016 [arXiv:1510.06266 [hep-ph]].

[19] E. Hernández and J. Nieves, Phys. Rev. D 95 (2017) no.5, 053007 doi:10.1103/PhysRevD.95.053007 [arXiv:1612.02343 [hep-ph]].

[20] J. E. Sobczyk, E. Hernández, S. X. Nakamura, J. Nieves and T. Sato, Phys. Rev. D 98 (2018) no.7, 073001 doi:10.1103/PhysRevD.98.073001 [arXiv:1807.11281 [hep-ph]].

[21] R. González-Jiménez, N. Jachowicz, K. Niewczas, J. Nys, V. Pandey, T. Van Cuyck and N. Van Dessel, Phys. Rev. D 95 (2017) no.11, 113007 doi:10.1103/PhysRevD.95.113007 [arXiv:1612.05511 [nucl-th]].

[22] D. Rein and L. M. Sehgal, Annals Phys. 133, 79 (1981). doi:10.1016/0003-4916(81)90242-6 
[23] J. Nieves, I. Ruiz Simo and M. J. Vicente Vacas, Phys. Rev. C 83 (2011) 045501 doi:10.1103/PhysRevC.83.045501 [arXiv:1102.2777 [hep-ph]].

[24] G. D. Megias, J. E. Amaro, M. B. Barbaro, J. A. Caballero, T. W. Donnelly and I. Ruiz Simo, Phys. Rev. D 94 (2016) no.9, 093004 doi:10.1103/PhysRevD.94.093004 [arXiv:1607.08565 [nucl-th]].

[25] N. Rocco, C. Barbieri, O. Benhar, A. De Pace and A. Lovato, Phys. Rev. C 99 (2019) no.2, 025502 doi:10.1103/PhysRevC.99.025502 [arXiv:1810.07647 [nucl-th]].

[26] https://gibuu.hepforge.org.

[27] E. C. Poggio, H. R. Quinn and S. Weinberg, Phys. Rev. D 13 (1976) 1958. doi:10.1103/PhysRevD.13.1958

[28] C. Andreopoulos et al., arXiv:1907.13252 [hep-ph]. 